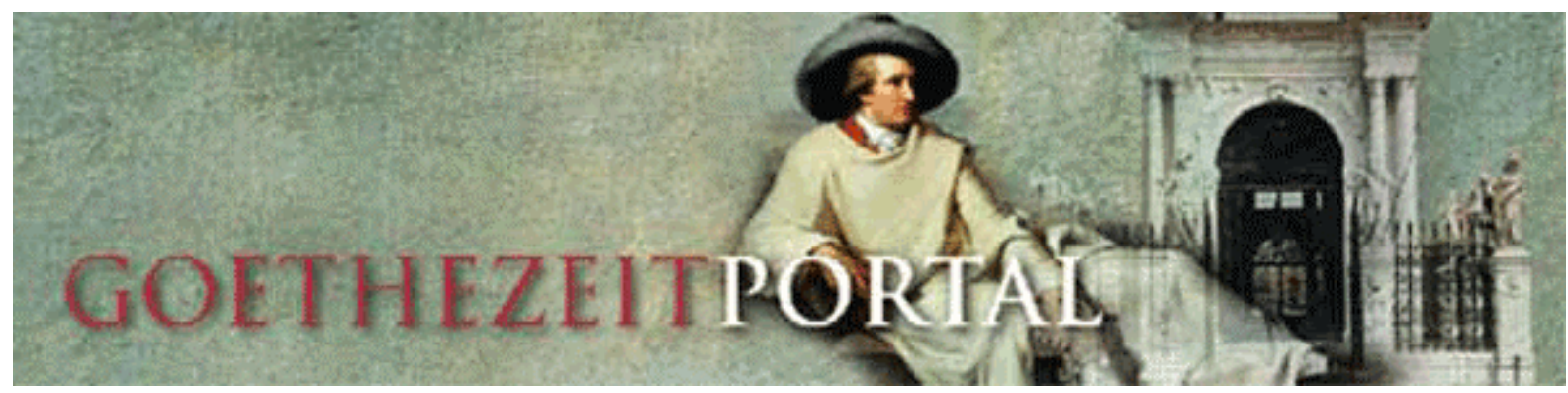

HENDRIK BIRUS

\title{
Lessing und die Weltliteratur
}

\author{
Vorblatt
}

\section{Publikation}

Vortrag, 39. Kamenzer Lessing-Tage, 15. 1. - 27. 2. 2000, Kleine Welt

- Große Welt ,Mittwoch, 9. 2. 2000.

Publikation im Goethezeitportal

Vorlage: Datei des Autors

URL: < http://www.goethezeitportal.de/db/wiss/lessing/birus_weltliteratur.pdf $>$

Eingestellt am 30.01.2004

\section{Autor}

Prof. Dr. Hendrik Birus

Ludwig-Maximilians-Universität München

Institut für Allgemeine und Vergleichende Literaturwissenschaft

(Komparatistik)

Schellingstr. 3

80799 München

Emailadresse: <h.birus@1rz.uni-muenchen.de>

\section{Empfohlene Zitierweise}

Beim Zitieren empfehlen wir hinter den Titel das Datum der Einstellung oder des letzten Updates und nach der URL-Angabe das Datum Ihres letzten Besuchs dieser Online-Adresse anzugeben:

Hendrik Birus: Lessing und die Weltliteratur (30.01.2004).

In: Goethezeitportal. URL:

$<$ http://www.goethezeitportal.de/db/wiss/lessing/birus_weltliteratur.pdf $>$

(Datum Ihres letzten Besuches). 
Birus: Lessing und die Weltliteratur, S.1

\title{
HENDRIK BIRUS
}

\section{Lessing und die Weltliteratur}

\author{
was ein Buch sei bekümmert mich immer weni- \\ ger; was es mir bringt, was es in mir aufregt, das \\ ist die Hauptsache. \\ Goethe an Zelter, 20. 2. 1828
}

\section{I}

Genau besehen hat dieser Titel etwas Anachronistisches; denn der Ausdruck Welttiteratur ist erst vom späten Goethe geprägt worden, der am 31. Januar 1827 gegenüber Eckermann erklärte: „National-Literatur will jetzt nicht viel sagen, die Epoche der Welt-Literatur ist an der Zeit und jeder muß jetzt dazu wirken, diese Epoche zu beschleunigen. “1 Für Lessing dagegen war die Epoche der Nationalliteratur durchaus an der Zeit gewesen; und er hat in Hamburg nicht wenig für den „,süße[n] Traum“² gewirkt, „,den Deutschen ein Nationaltheater zu verschaffen, da wir Deutsche noch keine Nation sind“. ${ }^{3}$ Gleichwohl operierte er als Autor wie als Kritiker schon früh und je später desto mehr im Horizont der Weltliteratur. Um diesen literarischen Ausgriff in die 'große Welt' besser zu verstehen, muß man sich zunächst seine ursprüngliche 'kleine Welt' vergegenwärtigen.

Lessings privaten Ausgangspunkt markiert der viel kolportierte Bericht seines Bruders Karl Gotthelf über die Entstehung von Christoph Gottlob Glymanns Gemälde (heute im Lessing-Museum Kamenz):

\footnotetext{
${ }^{1}$ Goethe zu Eckermann, 31. 1. 1827, in: Johann Wolfgang Goethe: Sämtliche Werke. Briefe, Tagebücher und Gespräche ['Frankfurter Ausgabe'], 40 Bde., hrsg. v. Friedmar Apel, Hendrik Birus [u. a.], Frankfurt/Main 1986-1999, hier: II. Abteilung, Bd. 12, S. 225 (künftig mit der Sigle FA sowie Abteilungs-, Band- und Seitenzahl).

${ }^{2}$ Hamburgische Dramaturgie, 101-104. Stück, in: Gotthold Ephraim Lessing: Werke und Briefe in zwölf Bänden, hrsg. v. Wilfried Barner [u. a.], Frankfurt/M. 1985ff., hier: Bd. 6, S. 690 (künftig mit der Sigle Lessing, FA sowie Band- und Seitenzahl).
} 
Als ein Maler ihn im fünften Jahre mit einem Bauer, in welchem ein Vogel saß, malen wollte, hatte dieser Vorschlag seine ganze kindische Mißbilligung. Mit einem großen, großen Haufen Bücher, sagte er, müssen Sie mich malen, oder ich mag lieber gar nicht gemalt sein. Der Maler that es, und wer das Gemälde sah, erfuhr diese Anekdote. Es war eben der Maler, der ihn nachher im Zeichnen unterrichtete, und ihm frühzeitig Geschmack an den bildenden Künsten beibrachte. Denn, wie Lessing oft erzählte, war er kein ganz schlechter Künstler, und besaß sogar etwas Kunstgelehrsamkeit. Wie er sich aber nach Kamenz verirrt hatte, weiß Gott! ${ }^{4}$

Das letztere mag sich auch hinsichtlich Lessings mancher fragen, der Sachsen für nichts als ein 'neues Bundesland' hält und die Lausitz nur als Kälte- oder Hitzeloch aus der Wetterkarte kennt. Doch tatsächlich hatte Sachsen damals eine zentrale Stellung im Deutschen Reich inne: und dies nicht nur geographisch, sondern auch politisch als einer der größten Territorialstaaten und durch seine Personalunion mit dem Königreich Polen (bis 1763). Womöglich noch wichtiger war aber seine kulturelle Dominanz im deutschen Sprachraum, wie sie etwa im 6. und 7. Buch von Goethes Dichtung und Wahrheit ${ }^{5}$ bezeugt wird. Denn nicht nur verkörperte das Obersächsische das ganze 18. Jahrhundert hindurch unangefochten die Sprachnorm des Hochdeutschen, sondern die Landesuniversität Leipzig war auch die prestigereichste Hochschule Deutschlands. Und wie Johann Christoph Gottsched als einer ihrer wichtigsten Professoren zugleich über Jahrzehnte als Literaturpapst in Deutschland galt, so eroberte sich Sachsen damals eine führende Stellung in der deutschen Literatur: man denke außer dem Ehepaar Gottsched nur an Autoren wie Gottlieb Wilhelm Rabener, Christian Fürchtegott Gellert, Johann Elias Schlegel, Friedrich Wilhelm Zachariae und Christian Felix Weiße oder an den Aufstieg der 'Sächsischen Typenkomödie'.

Der junge Lessing hat wesentlich zu dieser Schlüsselstellung Sachsens innerhalb der deutschen Aufklärungskultur beigetragen. Zunächst aber ist er

\footnotetext{
${ }^{3}$ Ebd. S. 684.

${ }^{4}$ K. G. Lessing: Gotthold Ephraim Lessings Leben [1793-95], hrsg. u. eingel. v. Otto F. Lachmann, Leipzig. o.J. (Reclams Universal Bibliothek, Bd. 2408/2409), S. 22.

${ }^{5}$ Goethe, FA I 14, bes. S. 262-337.
} 
durch sie überhaupt auf den Weg gebracht worden. Denn daß die Lausitz 1623 von Sachsen erworben worden war, hatte erst die Blüte ihres protestantischen Bildungssystems ermöglicht; und von ihr profitierte Lessing sowohl beim Privatunterricht im väterlichen Pfarrhaus wie beim Gymnasialunterricht in der Stadtschule Kamenz. Und wie der kometenhafte Aufstieg Klopstocks in der deutschen Literatur nicht ohne den exzellenten Sprachunterricht und die Stilübungen in Schulpforta möglich gewesen wäre, so war es auch eine wichtige Voraussetzung für Lessings ganze spätere Entwicklung, daß er mit zwölf Jahren auf die andere (seit der Reformationszeit bestehende) sächsische Fürstenschule zu St. Afra in Meißen aufgenommen wurde und hier so rasche Fortschritte machte, daß er sie schon nach fünf (statt sechs) Jahren verlassen konnte, nachdem der Rektor seinem Vater erklärt hatte: „Es ist ein Pferd, das doppeltes Futter haben muß. Die Lektiones, die andern zu schwer werden, sind ihm kinderleicht. Wir können ihn fast nicht mehr brauchen. ${ }^{\text {“6 }}$ Die Universität Leipzig aber, die er siebzehnjährig als stud. theol. bezog, verkörperte programmatisch das Bildungsideal der 'Polyhistorie' durch weithin berühmte Professoren wie Gottsched, Gellert, Crusius, Abraham Kästner, Johann August Ernesti und Johann Friedrich Christ, von denen nicht wenige auch in ihren Nebenstunden dichteten. Daneben war Leipzig der Standort einer der bedeutendsten Theatertruppen Deutschlands, deren Prinzipalin, Friederike Caroline Neuber, die klassizistische Theaterkonzeption Gottscheds in die Tat umzusetzen suchte.

War dies der unverwechselbare Entstehungskontext seiner dramatischen, poetischen und kritischen Jugendwerke, so wurde ihm diese kleine literarische Welt doch alsbald zu eng. Zunächst wechselte er zum Medizinstudium, verließ dann aber fluchtartig Leipzig, um sich (nach kurzem Aufenthalt in Wittenberg) Ende 1748 als freier Schriftsteller in Berlin niederzulassen: das heißt, sich als Theaterautor, Fabeldichter, Lyriker, Gelehrter, Herausgeber, Übersetzer und Rezensent durchzuschlagen. Gerade als Kritiker beschäftigte er

\footnotetext{
${ }^{6}$ K. G. Lessing: Gotthold Ephraim Lessings Leben, S. 28.
} 
Birus: Lessing und die Weltliteratur, S.4

sich dabei nahezu mit allen Sparten der aktuellen - keineswegs nur 'schönen' Literatur. So immens dabei sein Weltgewinn war, so überkam ihn doch auch zunehmend ein Ungenügen am bloß Zeitgenössischen, wie es Goethe mehr als ein halbes Jahrhundert später in der Divan-Strophe artikulieren sollte:

Wer nicht von dreytausend Jahren

Sich weiß Rechenschaft zu geben, Bleib im Dunkeln unerfahren, Mag von Tag zu Tage leben. ${ }^{7}$

Als Gegenmittel gegen die Enge dieser vermeintlich 'großen Welt' der deutschen Gegenwartsliteratur griff Lessing auf einen literarischen Fundus zurück, dessen Anfänge er sich in St. Afra erworben hatte:

Theophrastus, Plautus und Terenz waren meine Welt, die ich in dem engen Bezirke einer klostermäßigen Schule, mit aller Bequemlichkeit studierte - - Wie gerne wünschte ich mir diese Jahre zurück; die einzigen, in welchen ich glücklich gelebt habe. ${ }^{8}$

So publizierte Lessing gemeinsam mit seinem Vetter Christlob Mylius in den Beyträgen zur Historie und Aufnahme des Theaters (1750): Des Herrn von Voltaire Gedanken über die Trauer- und Lustspiele der Engländer, aus seinen Briefen über die Engländer übersetzt, in denen dieser freimütig bekennt: „Ich finde kein großes Vergnügen in Lesung des Plautus und Aristophanes; warum? Ich bin weder ein Grieche noch ein Römer. Die Feinheit der Scherze, die Anspielung, das Unerwartete, alles ist für einen Ausländer verloren. [...]“, und merkt dazu kritisch an:

Es ist in der Tat kein allzurühmliches Geständnis, welches der Herr von Voltaire hier tut. Man kann sicher daraus schließen, daß er weder den Plautus noch den Aristophanes gelesen hat. Ein Gelehrter kann sich eben sowohl zu einem Griechen oder Römer machen, als er etwa ein Deutscher oder ein Franzose ist. Hat man denn nicht Hülfsmittel genug, die Sitten, die Gebräuche und die Charaktere sowohl der Griechen als der Römer kennen zu lernen? Freilich, wer den Aristophanes, zum Exempel, lesen will, ohne eine genaue Kenntnis der damaligen Staatsver-

\footnotetext{
${ }^{7}$ Goethe, FA I 3, S. 59 („Und wer franzet oder brittet [...]“, v. 13-16).

${ }^{8}$ „Vorrede“ zu G. E. Lessings Schriften. Dritter Teil (1753), in: Gotthold Ephraim Lessing: Werke, 8 Bde., hrsg. v. Herbert G. Göpfert [u. a.], München 1970-1979, hier: Bd. 3, S. 521 525, bes. S. 522 (künftig mit der Sigle $H L$ zitiert, wo immer der entsprechende Band der 'Frankfurter Ausgabe' noch nicht erschienen ist).
} 
Birus: Lessing und die Weltliteratur, S.5

fassung in Athen zu haben, der wird ihn bald mit Verdruß aus den Händen legen. Allein es ist ja seine eigne Schuld. ${ }^{9}$

Deshalb heißt es in der „Vorrede“ der Beyträge programmatisch:

Was die Muster, die wir vorlegen wollen, anbelangt, so glauben wir uns in den Stand gesetzt zu haben, daß wir aus dem Griechischen und Lateinischen, aus dem Französischen, Italiänischen, Englischen, Spanischen und Holländischen unsern Lesern von uns übersetzte Stücke werden liefern können. Auf die erstern zwei wollen wir unsern Fleiß besonders wenden. Wir wollen zuweilen aus dem Sophokles, Euripides und Äschylus ein Stück übersetzen; wozu wir allezeit ein solches wählen wollen, das von den neuern Poeten ist nachgeahmet worden, oder von dessen Inhalte wenigstens ein ähnliches neueres Stück zu finden ist. Dieses wollen wir auch mit dem Aristophanes, Plautus, Terenz und dem tragischen Seneca tun. Wir wollen sie dabei selbst untereinander vergleichen, und zu bestimmen suchen, was Sophokles vor dem Euripides, dieser vor jenem, beide vor dem Äschylus, und dieser vor beiden eignes habe. Auf gleiche Art wollen wir mit dem Terenz und Plautus verfahren. Es soll uns nicht genug sein, ein Stück von ihnen zu übersetzen, wir wollen auch zeigen, worinne und wie Terenz den Plautus, und Plautus den Aristophanes nachahme. Wir wollen dabei mit allem Fleiße diejenigen Stücke und Stellen aufsuchen, welche die neuern Dichter von diesen geborgt haben. Wir werden daraus notwendig einsehen lernen, welches die wahre und falsche Art, nachzuahmen sei, und den Vorzug der Alten vor den Neuern, oder, in gewissen Stücken, dieser vor jenen, daraus feste setzen können. Von den Stücken der neuen Ausländer aber, werden wir nur solche übersetzen, die in Deutschland bisher am wenigsten sind bekannt gewesen, und die man als Muster in ihrer Art ansehen muß. Wir werden besonders unser Augenmerk auf das englische und spanische Theater richten. Shakespear, Dryden, Wicherley, Vanbrugh, Cibber, Congreve sind Dichter, die man fast bei uns nur dem Namen nach kennet, und gleichwohl verdienen sie unsere Hochachtung sowohl als die gepriesenen französischen Dichter. Eben so ist es mit dem Lopez de Vega, Augustin Moreto, Antonio de Mendosa, Francisco de Rojas, Fernando de Zarate, Juan Perez de Montalvan, Antonio de Avevedo, Francisco Gonsalez de Bustos und andern. Diese sind alle Männer, die zwar eben so große Fehler als Schönheiten haben, von denen aber ein vernünftiger Nachahmer sich sehr vieles zu Nutze machen kann. Doch wollen wir die Franzosen, Italiäner und Holländer nicht

\footnotetext{
${ }^{9}$ Lessing, FA I, S. 734f. - Noch in der Hamburgischen Dramaturgie (71. Stück) schreibt Lessing höhnisch: „Es scheinet nicht, daß der Herr von Voltaire, seit dem er aus der Klasse bei den Jesuiten gekommen, den Terenz viel wieder gelesen habe. Er spricht ganz so davon, als von einem alten Traume; es schwebt ihm nur noch so was davon im Gedächtnisse; und das schreibt er auf gut Glück so hin, unbekümmert, ob es gehauen oder gestochen ist.“ (Lessing, FA VI, S. 536f.)
} 
Birus: Lessing und die Weltliteratur, S.6

vergessen. $[\ldots]^{10}$

Hier bereits finden wir in nuce das strategische Arsenal von Lessings späteren kritischen Feldzügen versammelt. Ihr virtuelles Motto (von dem Lessing salopp bekennt: ,wo dieses Sprüchelchen steht, will mir nicht gleich beifallen“"11) stammt aus den Institutiones divinae des Kirchenvaters Lactantius Firmianus (gest. um 330): „Primus sapientiae gradus est, falsa intelligere; secundus, vera cognoscere“; wozu Lessing bemerkt:

Ein kritischer Schriftsteller, dünkt mich, richtet seine Methode auch am besten nach diesem Sprüchelchen ein. Er suche sich nur ersten jemanden, mit dem er streiten kann: so kömmt er nach und nach in die Materie, und das übrige findet sich. ${ }^{12}$

Am Anfang steht also die Infragestellung weithin unangezweifelter Autoritäten - hier Voltaire; andernorts Gottsched, wenn etwa der 17. der Briefe, die neueste Litteratur betreffend (1759) mit den (ausgerechnet gegen seinen Freund Friedrich Nicolai gerichteten) Worten beginnt:

„Niemand“, sagen die Verfasser der Bibliothek [d. i. Bibliothek der schönen Wissenschaften und der freyen Künste], ,wird leugnen, daß die deutsche Schaubühne einen großen Teil ihrer ersten Verbesserungen dem Herrn Professor Gottsched zu danken habe."

Ich bin dieser Niemand; ich leugne es gerade zu. Es wäre zu wünschen, daß sich Herr Gottsched niemals mit dem Theater vermengt hätte. Seine vermeinten Verbesserungen betreffen entweder entbehrliche Kleinigkeiten, oder sind wahre Verschlimmerungen. ${ }^{13}$

Oder de La Motte, Richer, Breitinger und Batteux in den Abhandlungen über die Fabel (1759), Caylus, ja selbst Winckelmann im Laokoon (1766), Klotz in den Briefen, Antiquarischen Inhalts (1768/69). Diese Aufkündigung fragwürdiger zeitgenössischer Kanonisierungen fußt bei Lessing stets auf einem radikalen Blickwechsel von der Binnenperspektive der vor allem am französischen Klassizismus ausgerichteten deutsche Kunst- und Literaturszene zu einem die Sprachen und Kulturen Europas und des Vorderen Orients von den ersten An-

\footnotetext{
${ }^{10}$ Ebd. S. 728f.

${ }^{11}$ Lessing, FA VI, S. 535 (Hamburgische Dramaturgie, 70. Stück).

${ }^{12}$ Ebd.

${ }^{13}$ Lessing, FA IV, S. 499.
} 
Birus: Lessing und die Weltliteratur, S.7

fängen bis zur unmittelbaren Gegenwart umspannenden weltbürgerlichen Horizont. Und dies in doppelter Hinsicht: Einerseits werden die angemaßten $\mathrm{Au}-$ toritäten durch die wahren Originale ersetzt - hier die Epigonen der Tragédie classique durch die antiken Tragödien- und Komödienautoren oder im Vade mecum für den Hrn. Sam. Gotth. Lange (1753) und in den Rettungen des Horaz (1754) der inkompetente Nachahmer durch sein verschandeltes Vorbild, oder man denke nur (in einer Rezension von Michael Conrad Curtius' Übersetzung [1753]) an die Apostrophierung der Poetik des Aristoteles als „der Quell aus welchem alle Horaze, alle Boileaus, alle Hedelins, alle Bodmers, bis so gar auf die Gottschede ihre Fluren bewässert haben“. ${ }^{14}$ Andererseits sucht Lessing nach den legitimen Nachfahren solcher prototypischen Werke und Autoren wie hier das englische und spanische Theater als Alternative zum französischen oder Shakespeare statt Voltaire oder als überragenden Zeitgenossen Diderot, da sich „,nach dem Aristoteles, kein philosophischerer Geist mit dem Theater abgegeben hat, als Er. Daher sieht er auch die Bühne seiner Nation bei weitem auf der Stufe der Vollkommenheit nicht, auf welcher sie, unter uns die schalen Köpfe erblicken, an deren Spitze der Prof. Gottsched ist.“15

Gerade ein solches Bewußtsein eigener Defizite gegenüber ausländischen Vorbildern der Vergangenheit und Gegenwart war aber für Lessing alles andere als lähmend, sondern vielmehr eine ständige Herausforderung. Und dies einerseits ganz persönlich; hatte er doch bereits als Zwanzigjähriger seinem Vater geschrieben:

Wenn man mir mit Recht den Titel eines deutschen Moliere beilegen könnte, so könnte ich gewiß eines ewigen Namens versichert sein. Die Wahrheit zu gestehen, so habe ich zwar große Lust ihn zu verdienen, aber sein Umfang und meine Ohnmacht sind zwei Stücke die auch die größte Lust erstücken könnten. ${ }^{16}$

Daß dieser Ehrgeiz andererseits auf eine anderen Völkern ebenbürtige deutsche Nationalliteratur zielte, zeigt Lessings Gedankenexperiment im 7. Literatur-

\footnotetext{
${ }_{15}^{14}$ Lessing, FA II, S. 532.

15 „Vorrede des Übersetzers“ zu Das Theater des Herrn Diderot. Aus dem Französischen (1760), in: Lessing, FA 5/1, S. 13.
} 
brief (1759): Was wäre, „wenn durch eine große, wunderbare Weltveränderung auf einmal alle Bücher, die deutsch geschriebenen ausgenommen, untergingen“"? Worauf er hinsichtlich der 'schönen Wissenschaften' in Deutschland die so selbstbewußte wie ironisch überzogene Perspektive entwirft:

Nein, auch in jenen fehlt es uns nicht an Männern, die alsdenn an die Stelle der großen Ausländer, und der noch größern Alten treten müßten und könnten! Klopstock würde Homer; Cramer, Pindar; Utz, Horatz; Gleim, Anakreon; Gessner, Theokrit; Wieland, Lukrez - ${ }^{17}$

Dies erinnert nicht von ungefähr an jene seit dem Humanismus gängigen und nicht selten die Grenzen zum Lächerlichen überschreitenden Apostrophierungen, etwa eines Rachel als „deutscher Juvenal“ oder der Karschin als „deutsche Sappho“; kein Wunder also, daß Lessing in dieser Aufzählung keinen einzigen deutschen Dramenautor nennt. Doch schon in der (bereits zitierten) „Vorrede“ der Beyträge zur Historie und Aufnahme des Theaters (1750) hatte es programmatisch geheißen:

Wir haben zu wenig eigne Stücke; und den meisten dieser Stücke merkt man das Ausländische allzusehr an. Der sicherste Charakter also, den man daraus von dem Deutschen wird bestimmen können, ist, daß er überall das Gute, wo er es findet, billige, und es sich zu Nutze mache. Das ist gewiß, wollte der Deutsche in der dramatischen Poesie seinem eignen Naturelle folgen, so würde unsre Schaubühne mehr der englischen als französischen gleichen. ${ }^{18}$

Erscheint dies hier noch vergleichsweise zurückhaltend artikuliert, so hat Lessing in dem berühmten 17. Literaturbrief (1759) ein schonungsloses Bild der von ihm vorgefundenen Situation des deutschen Theaters und seiner Abhängigkeit von Frankreich gezeichnet:

Als die Neuberin blühte, und so mancher den Beruf fühlte, sich um sie und die Bühne verdient zu machen, sahe es freilich mit unserer dramatischen Poesie sehr elend aus. Man kannte keine Regeln; man bekümmerte sich um keine Muster. Unsre Staats- und Helden-Actionen waren voller Unsinn, Bombast, Schmutz und Pöbelwitz. Unsre Lustspiele bestanden in Verkleidungen und Zaubereien; und Prügel waren die witzigsten Einfälle derselben. Dieses Verderbnis einzusehen, brauchte man

\footnotetext{
${ }^{16}$ Lessing, FA XI/1, S. 24 (an Johann Gottfried Lessing, 28. 4. 1749).

${ }^{17}$ Lessing, FA IV, S. 467f.

${ }^{18}$ Lessing, FA I, S. 729.
} 
Birus: Lessing und die Weltliteratur, S.9

eben nicht der feinste und größte Geist zu sein. Auch war Herr Gottsched nicht der erste, der es einsahe; er war nur der erste, der sich Kräfte genug zutraute, ihm abzuhelfen. Und wie ging er damit zu Werke? Er verstand ein wenig Französisch und fing an zu übersetzen, er ermunterte alles, was reimen und Oui Monsieur verstehen konnte, gleichfalls zu übersetzen; er verfertigte, wie ein Schweitzerischer Kunstrichter sagt, mit Kleister und Schere seinen Cato; er ließ den Darius und die Austern, die Elise und den Bock im Processe, den Aurelius und den Wizling, die Banise und den Hypocondristen ohne Kleister und Schere machen; er legte seinen Fluch auf das extemporieren; er ließ den Harlequin feierlich vom Theater vertreiben, welches selbst die größte Harlequinade war, die jemals gespielt worden; kurz, er wollte nicht sowohl unser altes Theater verbessern, als der Schöpfer eines ganz neuen sein. Und was für eines neuen? Eines Französierenden; ohne zu untersuchen, ob dieses französierende Theater der deutschen Denkungsart angemessen sei, oder nicht. ${ }^{19}$

Und Lessing leitet daraus - mit unabsehbaren Konsequenzen für die Shakespeare-Rezeption des Sturm und Drang und der Romantik - die alternative Konzeption $\mathrm{ab}$,

Er [d.i. Gottsched] hätte aus unsern alten dramatische Stücken, welche er vertrieb, hinlänglich abmerken können, daß wir mehr in den Geschmack der Engländer, als der Franzosen einschlagen [...]. Er hätte also auf dieser Spur bleiben sollen, und sie würde ihn geraden Weges auf das englische Theater geführet haben. - [...] Wenn man die Meisterstücke des Shakespear, mit einigen bescheidenen Veränderungen, unsern Deutschen übersetzt hätte, ich weiß gewiß, es würde von bessern Folgen gewesen sein, als daß man sie mit dem Corneille und Racine so bekannt gemacht hat. [...] Auch nach den Mustern der Alten die Sache zu entscheiden, ist Shakespear ein weit größerer tragischer Dichter als Corneille; obgleich dieser die Alten sehr wohl, und jener fast gar nicht gekannt hat. Corneille kömmt ihnen in der mechanischen Einrichtung, und Shakespear in dem Wesentlichen näher. Der Engländer erreicht den Zweck der Tragödie fast immer, so sonderbare und ihm eigene Wege er auch wählet; und der Franzose erreicht ihn fast niemals, ob er gleich die gebahnten Wege der Alten betritt. ${ }^{20}$

Wie sehr aber der hier für das deutsche Theater proklamierte Paradigmenwechsel von der französischen zur englischen Tragödie einem unmittelbaren produktiven Interesse Lessings entsprang, zeigt die anschließende Mitteilung sei-

\footnotetext{
${ }^{19}$ Lessing, FA IV, S. 499f.

${ }^{20}$ Ebd. S. 500f.
} 
nes eigenen anonymen Faust-Fragments, die er mit den Worten beschließt: „Was sagen Sie zu dieser Scene? Sie wünschen ein deutsches Stück, das lauter solche Scenen hätte? Ich auch!“21 Kein Wunder, daß ihn ein gutes Jahrzehnt später ein Freund „O Shakespear-Lessing! ‘22 anredet.

$\mathrm{Da}$ diese den eingespielten Kanon in Frage stellende Tendenz in Lessings Hamburgischer Dramaturgie (1767-1769) kulminierte, lag gewiß nicht am Repertoire des Hamburger Theaters, als dessen Rezensionsorgan sie dienen sollte; denn dieses umfaßte überwiegend Übersetzungen französischer Stücke und ihre deutschen Nachahmungen. Lessing schreibt deshalb kurz vor Schluß resigniert:

Wir sind noch immer die geschwornen Nachahmer alles Ausländischen, besonders noch immer die untertänigen Bewunderer der nie genug bewunderten Franzosen; alles was uns von jenseit dem Rheine kömmt, ist schön, reizend, allerliebst, göttlich; lieber verleugnen wir Gesicht und Gehör, als daß wir es anders finden sollten; lieber wollen wir Plumpheit für Ungezwungenheit, Frechheit für Grazie, Grimasse für Ausdruck, ein Geklingle von Reimen für Poesie, Geheule für Musik, uns einreden lassen, als im geringsten an der Superiorität zweifeln, welche dieses liebenswürdige Volk, dieses erste Volk in der Welt, wie es sich selbst sehr bescheiden zu nennen pflegt, in allem, was gut und schön und erhaben und anständig ist, von dem gerechten Schicksale zu seinem Anteile erhalten hat. ${ }^{23}$

Doch Lessing ergreift immer wieder die Gelegenheit, diese 'kleine Welt' des Hamburger Spielplans in umfassendere Perspektiven zu rücken; wenn er etwa gleich in den beiden ersten Stücken ${ }^{24}$ Johann Friedrich von Cronegks Trauerspiel Olint und Sophronia (postum 1760) mit der entsprechenden Episode aus Tassos Gerusalemme liberata vergleicht und dies zu einer prinzipiellen Kritik des 'christlichen Trauerspiels' steigert oder wenn er die Besprechungen von Voltaires Semiramis (1748) und Zä̈re $(1732)^{25}$ zum Anlaß vernichtender Vergleiche mit Shakespeares Hamlet, Romeo und Julia und Hamlet nimmt oder

\footnotetext{
${ }^{21}$ Ebd. S. 501.

${ }^{22}$ Johann Arnold Ebert an Lessing, 14. 3. 1772 (Lessing, FA XI/1, S. 377).

${ }^{23}$ Lessing, FA VI, S. 684f. (101.-104. Stück).

${ }^{24}$ Ebd. S. 187-196.

${ }^{25}$ Ebd. S. 234-242 u. 255-265 (10.-12. u. 15.-16. Stück).
} 
wenn er Thomas Corneilles Graf von Essex (1678) bis ins Detail - nicht eben zu seinen Gunsten - mit anderen englischen und spanischen Gestaltungen dieses Stoffs und mit Diderots (früher von ihm selbst übersetzter) Dramentheorie konfrontiert. ${ }^{26}$ So erweitert Lessing eine Besprechung von Voltaires Mérope (1737) zunächst zu einer Vergleichung mit Maffeis gleichnamiger Tragödie (1713), steigert diese dann aber - an Hand der knappen Nacherzählung in der 184. Fabel des Hyginus - zu einer 'Rettung' des Euripides und seiner nur noch in wenigen Fragmenten überlieferten stoffgleichen Tragödie Chresphontes; andererseits referiert er hier ausführlich die mehr oder weniger verdeckt polemischen Sendschreiben Voltaires und Maffeis (wobei er zu Recht Voltaire hinter dem Namen de la Lindelle vermutet), sowie die Einlassungen des Jesuitenpaters Tournemine und des Aristoteles-Übersetzers Dacier, um endlich eine Klärung der von ihnen erörterten Probleme im Blick auf die Poetik des Aristoteles und den ,besten französischen Kunstrichter“ Diderot ${ }^{27} \mathrm{zu}$ unternehmen. ${ }^{28} \mathrm{Ja}$, in seiner Besprechung von Christian Felix Weißes Tragödie Richard der Dritte $(1759 / 1765)^{29}$ entfaltet Lessing vor dem Hintergrund von Shakespeares gleichnamigem Stück und unter Berufung auf Aristoteles wie in Abwehr Corneilles die Grundzüge seiner eigenen Tragödientheorie und nutzt schließlich die Besprechung von Diderots Der Hausvater ${ }^{30}$ zur Konfrontation von dessen Dramentheorie mit der seiner französischen Zeitgenossen, dann aber vor allem mit der des Aristoteles.

II

Rühmt man an Lessing, daß er wie keiner seiner Zeitgenossen in Deutschland (mit Goethe zu sprechen) die Epoche der Welt-Literatur befördert habe, so bedarf der Begriff der Weltliteratur einer Präzisierung. Denn versteht man ihn bloß quantitativ als Summe der verschiedenen Literaturen und literarischen

\footnotetext{
${ }^{26}$ Ebd. S. 291-307 u. 450-535 (22.-25. u. 54.-70. Stück).

${ }^{27}$ Ebd. S. 419.

${ }^{28}$ Ebd. S. 359-433 (36.-51. Stück).

${ }^{29}$ Ebd. S. 548-597 (73.-83. Stück).
} 
Werke der Welt, so hat Lessing gewiß mit einer ganzen Anzahl von Titeln der spätantiken, mittelalterlichen, spanischen und englischen Literatur zur Erweiterung des literarischen Horizonts des deutschen Publikums beigetragen. Doch seine ungleich gewichtigeren Beiträge zu Homer, den antiken Dramatikern, Lyrikern und Fabeldichtern, ja selbst zu Shakespeare oder Diderot hatten nicht den Charakter von Neuentdeckungen, wie er andererseits an literarischem Spürsinn für die verschiedensten Regionen der Weltkultur durch seinen (nur 15 Jahre jüngeren) Zeitgenossen Herder, ja selbst durch Goethe weit übertroffen wurde. Versteht man aber unter 'Weltliteratur' den weltumspannenden Kanon der bedeutendsten literarischen Werke, so vermag allenfalls Nathan der Weise als erstes Werk der neueren deutschen Literatur Anspruch auf eine solche Weltgeltung zu erheben, ohne damit Lessing in ähnlichem Maße zu einem deutschen Autor der Weltliteratur zu machen wie nach ihm Goethe, Schiller, Hölderlin, E.T.A. Hoffmann oder Heine, ganz zu schweigen von Th. Mann, Musil, Kafka oder Celan.

So wenig aber solche Momente völlig aus ihm auszuscheiden sind, so hatte doch Goethe bei der Prägung des Begriffs 'Weltliteratur' etwas grundsätzlich anderes als einen quantitativen oder qualitativen Ordnungszusammenhang literarischer Werke im Blick: nämlich eine durch die Fortschritte auf den Gebieten der Produktion, des Handels, des Verkehrs und der Kommunikation (modern gesprochen: den Prozeß der Globalisierung) ermöglichte neue Form der internationalen literarischen Wechselwirkungen ${ }^{31}$ :

$\mathrm{Zu}$ einer Zeit wo die Eilboten aller Art aus allen Weltgegenden her immerfort sich kreuzen, ist einem jeden Strebsamen höchst nöthig seine Stellung gegen die eigne Nation und gegen die übrigen kennen zu lernen. Deßhalb findet ein denkender Literator alle Ursache jede Kleinkrämerey aufzugeben und sich in der großen Welt des Handelns

\footnotetext{
${ }^{30}$ Ebd. S. 599-655 (84.-95. Stück).

${ }^{31}$ Vgl. Hendrik Birus: Goethes Idee der Weltiteratur. Eine historische Vergegenwärtigung, in: Weltliteratur heute. Konzepte und Perspektiven, hrsg. v. Manfred Schmeling, Würzburg 1995 (Saarbrücker Beiträge zur Vergleichenden Literatur- und Kulturwissenschaft, Bd. 1), S. 5-28; u. ders.: Am Schnittpunkt von Komparatistik und Germanistik: Die Idee der Weltliteratur, in: Germanistik und Komparatistik. DFG-Symposion 1993, hrsg. v. H. Birus, Stuttgart, Weimar 1995 (Germanistische Symposien, Berichtsbd. 16), S. 439-457.
} 
umzusehen. $^{32}$

Zwar hatte Goethe bereits 1800 in den Propyläen für Kunst und Wissenschaft eine ,allgemeine, freie Wechselwirkung aller zugleich Lebenden, in steter Rücksicht auf das was uns vom Vergangenen übrig und bekannt ist" ${ }^{33}$ gefordert. Und er schrieb am 10. 6. 1822 an den Grafen Reinhard:

Auch von auswärts ereignet sich mir Wünschenswertes; die Franzosen übersetzen meine dramatischen Arbeiten und ich muß eine Befreiung von Vorurteil, eine Höhe ihrer Ansicht bewundern. Indessen die Deutschen in einer beinahe unverständlich werdenden Sprache sich Gedanken und Urteil einander mitteilen, so bedient sich der Franzose herkömmlicher Ausdrücke, weiß sie aber so zu stellen, daß sie wie ein aus Planspiegeln zusammengesetzter Hohlspiegel kräftig auf einen Focus zusammen wirken.

In England hat ein Herr Soane meinen Faust bewundernswürdig verstanden und dessen Eigentümlichkeiten mit den Eigentümlichkeiten seiner Sprache und den Forderungen seiner Nation in Harmonie zu bringen gewußt; ich besitze die ersten Bogen mit nebengedrucktem Original. Überhaupt will mir bedünken, daß die Nationen sich unter einander mehr als je verstehen lernen; die Mißverständnisse scheinen nur innerhalb des eigenen Körpers einer jeden zu liegen. ${ }^{34}$

Doch programmatisch realisierte Goethe schließlich einen solchen internationalen Austausch zwischen den „Litteraturen und Litteratoren“ (Tagebuch, 8. 5. 1826) unter dem Titel 'Weltliteratur' in den letzten Heften seiner Zeitschrift Ueber Kunst und Alterthum (1816-32) - und zwar dank der Überzeugung: „es bilde sich eine allgemeine Weltliteratur, worin uns Deutschen eine ehrenvolle Rolle vorbehalten ist“. 35

Davon konnte zu Lessings Lebzeiten noch keine Rede sein, der sich in seiner Jugend von Voltaire geringschätzig behandelt fühlte und dem später bei seiner Bewerbung um die Berliner Bibliothekarsstelle ein unbedeutender französischer Schriftsteller vorgezogen wurde, den man auch noch mit einem andern

\footnotetext{
${ }^{32}$ Goethe, FA I 22, S. 280 (Notice sur la vie et les Ouvrages de Goethe par Albert Stapfer).

${ }^{33}$ Goethe, FA I 18, S. 809 (Die Preisaufgabe betreffend).

${ }^{34}$ Goethe, FA II 9, S. 258.

${ }^{35}$ Goethe, FA I 22, S. 356 (Le Tasse, drame historique en cinq actes, par M. Alexander Duval).
} 
verwechselt hatte. ${ }^{36}$ War doch Bouhours' ,abgeschmackte Frage“, ${ }^{37}$ ob ein Deutscher, angesichts der Roheit seiner Sprache, überhaupt 'bel esprit' haben könne, noch 1740 von einem Braunschweiger Lehrer entschieden verneint worden. ${ }^{38}$ Daher erwähnt es Lessing ausdrücklich im 14. Stück der Hamburgischen Dramaturgie, daß mehr als sechs Jahre zuvor im Journal étranger (Dezember 1761) eine positive Kritik seiner Miß Sara Sampson (1755) erschienen sei: „Das bürgerliche Trauerspiel hat an dem französischen Kunstrichter, welcher die Sara seiner Nation bekannt gemacht, einen sehr gründlichen Verteidiger gefunden. Die Franzosen billigen sonst selten etwas, wovon sie kein Muster unter sich haben. ${ }^{39} \mathrm{Ja}$, er schmeichelte sich sogar mit der Vermutung, jene Kritik stamme von keinem Geringeren als Diderot. Und entsprechend frappiert berichtete ihm sein Freund Friedrich Nicolai am 26. 4. 1773:

Ich habe neulich einen Brief von Cacault an Mr. Bitaubé gelesen, worin er ihm schrieb: ,je vous prie, ayez la patience de lire la Dramaturgie; mais ayez la patience de la lire à l'allemande, c'est à dire, de tout examiner en lisant." Eines so großen Wunders hätte ich mich doch nicht versehen. Ein Franzose soll dem andern die Dramaturgie anpreisen! Nun glaube ich fast, daß'es möglich ist, Franz von Sickingen von Döbbelin auf dem französischen Theater zu Paris aufführen zu sehen! ${ }^{40}$

Ja, Nicolai wußte gar am 24. 4. 1777 zu vermelden:

Cacault schreibt mir unter andern: Herr Mercier wolle die Dramaturgie französisch herausgeben, und damit, vereinigt mit seinen Anmerkungen, alle französischen Tragödienschreiber niederschlagen. Nochmals

\footnotetext{
${ }^{36}$ Vgl. Erich Schmidt: Lessing. Geschichte seines Lebens und seiner Schriften, 2 Bde., 4. durchges. Aufl., Berlin 1923, hier: Bd. 1, S. 524-528.

${ }^{37}$ Lessing (Rez.): [Boulenger de Rivery:] Fables et Contes (1754) (HL III, S. 239); die Frage findet sich im vierten von Dominique Bouhours' Entretiens d'Ariste et d'Eugène (1671). Noch in der Hamburgischen Dramaturgie (81. Stück) heißt es lapidar, Bouhours habe sich mit seiner Frage ,lächerlich gemacht“(Lessing, FA VI, S. 585).

${ }^{38} \mathrm{Vgl}$. Eleazar de Mauvillon, Lettres françaises et germaniques (Nr. 11); sowie hierzu E. Schmidt, Lessing, Bd. 1, S. 58.

${ }^{39}$ Lessing, FA VI, S. 250f. (14. Stück).

40 „Ich bitte Sie, haben Sie die Geduld, die Dramaturgie zu lesen; aber haben Sie die Geduld, sie deutsche zu lesen, das heißt, beim Lesen genau zu prüfen“ (Lessing, FA XI/2, S. 546 u. 1033). Schon am 16. 1. 1773 hatte Lessings Bruder Karl berichtet: „Es ist eine Rarität über alle Raritäten: Cacault, ein Professor de Ecole militaire in Paris, reiset seit etlichen Jahren herum, um andere Menschen, als Franzosen kennen zu lernen, und legt sich gar auf die deutsche Litteratur. An Ramlers Oden hat er so viel Geschmack gefunden, daß er sie übersetzt hat, und die Übersetzung itzt unter Ramlers Aufsicht verbessert und ausfeilt. In Paris sollen sie gedruckt, und unserm Könige dediciert werden.“ (Ebd. S. 502.)
} 
Birus: Lessing und die Weltliteratur, S.15

Glück zu! ${ }^{41}$

Tatsächlich hat François Cacault (1742-1805) nach Lessings Tod eine französische Übersetzung der Hamburgischen Dramaturgie herausgebracht: Dramaturgie ou observations critiques sur plusieurs pièces de théâtre, traduite de l'allemand de Lessing par un François et publiée par M. J. (Paris 1785).

Wie grundlegend hatte sich - nicht zuletzt dank Lessings Wirken - innerhalb weniger Jahrzehnte die internationale Stellung der deutschen Literatur verändert, so daß Goethe im Zusammenhang des 'Weltliteratur'-Hefts VI/1 von Ueber Kunst und Alterthum und speziell seines Interesses an der „,innern Bedeutung“ der „Aufmerksamkeit der Franzosen auf uns“ seinem Verleger Cotta am 26. 1. 1827 schrieb: „Auf die ausländische Literatur muß man besonders jetzt hinweisen, da jene sich um uns zu bekümmern anfangen“; 42 denn: „Eine jede Literatur ennüyirt sich zuletzt in sich selbst, wenn sie nicht durch fremde Theilnahme wieder aufgefrischt wird. “43 Ja, Goethe benennt ausdrücklich diesen historischen Kontrast, wenn er anläßlich einer französischen Übersetzung seiner dramatischen Werke davon spricht, daß es ,,angenehm sey zu erfahren, wie sich seine Bemühungen einer Nachbarnation darstellen, welche von jeher nur im Allgemeinen an deutschem Bestreben Theil genommen, weniges davon gekannt, das wenigste gebilligt hat", und fortfährt:

Nun dürfen wir nicht läugnen, daß wir Deutsche gerade wegen dieses eigensinnigen Ablehnens auch gegen sie eine entschiedene Abneigung empfunden, daß wir uns um ihr Urtheil wenig bekümmert und sie gegenseitig nicht zum günstigsten beurtheilt haben. Merkwürdig jedoch mußte es uns in der neusten Zeit werden, wenn dasjenige was wir an uns selbst schätzten, auch von ihnen anfing geschätzt zu werden, und zwar nicht, wie bisher, von einzelnen besonders gewogenen Personen, sondern in einem sich immer weiter ausbreitenden Kreise.

[...] Und so darf uns denn in weltbürgerlichem Sinne wohl freuen, daß ein durch so viel Prüfungs- und Läuterungsepochen durchgegangenes Volk sich nach frischen Quellen umsieht um sich zu erquicken, zu stär-

\footnotetext{
${ }^{41}$ Lessing, FA XII, S. 68.

${ }^{42}$ Goethe: Werke, 133 Bde., hg. im Auftrage d. Großherzogin Sophie von Sachsen ['Weimarer Ausgabe'], Weimar 1887-1919 (Reprint München 1987), hier: IV. Abtheilung, Bd. 42, S. $26 f$.

${ }^{43}$ Goethe, FA I 22, S. 428 (Bezüge nach Außen).
} 
ken, herzustellen, und sich deßhalb mehr als jemals nach außen, zwar nicht zu einem vollendeten, anerkannten, sondern zu einem lebendigen, selbst noch im Streben und Streiten begriffenen Nachbarvolke hinwendet.

Aber nicht allein auf den Deutschen richten sie ihre Aufmerksamkeit, sondern auch auf den Engländer, den Italiäner; und wenn sie Schiller's Kabale und Liebe in drey Nach- und Umbildungen gleichzeitig auf drey Theatern günstig aufnehmen; wenn sie Musäus Mährchen übersetzen: so sind Lord Byron, Walter Scott, und Cooper bey ihnen gleichfalls einheimisch, und sie wissen die Verdienste Manzoni's nach Gebühr zu würdigen.

Ja wenn man genau auf den Gang den sie nehmen Acht giebt, so möchte die Zeit herannahen, wo sie uns Deutsche an gründlich freysinniger Kritik zu übertreffen auf den Weg gelangen. Möge sich dieß ein jeder, den es angeht, gesagt seyn lassen. ${ }^{4}$

Hatte Lessing - freilich um der Ebenbürtigkeit der deutschen Nationalliteratur willen - mit seinen literaturkritischen Schriften die Fundamente für die von Goethe proklamierte „Epoche der Welt-Literatur“ gelegt, so lieferte er zugleich mit seinem letzten Drama Nathan der Weise seinen wichtigsten Beitrag zu einer so verstandenen 'Weltliteratur': als einer die Grenzen der Nationen, der Kulturen und der Diskursgemeinschaften überschreitenden und zwischen ihnen vermittelnden literarischen Kommunikation in weltbürgerlicher Absicht. Denn als ihm im Sommer 1778 die Zensurfreiheit nicht nur für die ReimarusFragmente, sondern auch für „seine sogenannten Antigözzischen Blätter“ entzogen wurde, ${ }^{45}$ ja ihm sogar untersagt wurde, ,daß er in Religions-Sachen, so wenig hier als auswärts, auch weder unter seinem noch anderen angenommenen Namen, ohne vorherige Genehmigung des Fürst. Geheimen Ministerii ferner etwas drucken lassen möge““ 46 schrieb er an Elise Reimarus: „Ich muß versuchen, ob man mich auf meiner alten Kanzel, auf dem Theater wenigstens, noch ungestört will predigen lassen. “47 So machte er sich also daran, die Gattung des Dramas zum Medium der ihm sonst verwehrten öffentlichen Kommu-

\footnotetext{
${ }^{44}$ Ebd. S. 258-260 (Oeuvres dramatiques de Goethe, traduites de l'allemand; prédédées d'une Notice biographique et litéraire. 4 voll. in 8$)$.

${ }^{45}$ Vgl. Herzog Karls von Braunschweig Briefe vom 13. 7. u. 3. 8. 1778, in: Lessing, FA XII, S. 162-164, 179f.

${ }^{46}$. Herzog Karl von Braunschweig, 17. 8. 1778, in: ebd. S. 186f.

${ }^{47}$ Ebd. S. 193 (6. 9. 1778).
} 
nikation (Brechtisch gesprochen) 'umzufunktionieren', um so „dem Feinde auf einer andern Seite damit in die Flanke [zu] fallen“. ${ }^{48}$ Als Vehikel hierfür bediente er sich des Prototyps einer der wichtigsten Gattungen der europäischen Erzählliteratur: Boccaccios Novellensammlung Il Decameron (1349-53). Seinem Bruder Karl gegenüber spielte er dies als „,närrischen Einfall“ der vergangenen Nacht herunter, fügte aber ernsthaft hinzu:

Ich möchte zwar nicht gern, daß der eigentliche Inhalt meines anzukündigenden Stücks allzufrüh bekannt würde; aber doch, wenn Ihr, Du oder Moses, ihn wissen wollt, so schlagt das Decamerone des Bocaccio auf: Giornata I. Nov. III. Melchisedech Giudeo. Ich glaube, eine sehr interessante Episode dazu erfunden zu haben, daß sich alles sehr gut soll lesen lassen, und ich gewiß den Theologen einen ärgern Possen damit spielen will, als noch mit zehn Fragmenten. ${ }^{49}$

Allerdings warnte er seinen Bruder Karl - und mit ihm die Berliner Aufklärer davor, sich „eine ganz unrechte Idee davon“ zu machen: „Es wird nichts weniger, als ein satirisches Stück, um den Kampfplatz mit Hohngelächter zu verlassen. Es wird ein so rührendes Stück, als ich nur immer gemacht habe““. ${ }^{50} \mathrm{Ja}$, er steigerte seinen Kunstcharakter noch, indem er es - wie zuvor nur einige Dramenfragmente - in Blankverse brachte, wie sie Shakespeare und seine Nachfolger benutzten und die mit Lessings Nathan der Weise zum kanonischen Metrum des deutschen Versdramas von Goethe und Schiller bis zum späten Hauptmann wurden. Freilich spielte er den damit markierten Kunstanspruch gleich wieder herunter, indem er seinem Bruder schrieb: „Meine Prose hat mir von jeher mehr Zeit gekostet, als Verse. Ja, wirst Du sagen, aber solche Verse! - Mit Erlaubnis; ich dächte, sie wären viel schlechter, wenn sie viel besser wären." ${ }^{\text {"51 }}$ So betonte er auch gegenüber einem Dichterkollegen:

[...] ich habe wirklich die Verse nicht des Wohlklanges wegen gewählt: sondern weil ich glaubte, daß der orientalische Ton, den ich doch hier

\footnotetext{
${ }^{48}$ An Karl Lessing, 7. 11. 1778 (ebd. S. 207).

${ }^{49}$ Ebd. S. 186 (11. 8. 1778); entsprechend zu Elise Reimarus, 6. 9.1778 (ebd. S. 193).

${ }^{50}$ Ebd. S. 200 (20. 10. 1778).

${ }^{51}$ Ebd. S. 209 (7. 12. 1778). Der Gestus dieser Briefstelle erinnert mich immer an den Anfang von Brechts Flüchtlingsgesprächen: „Das Bier ist kein Bier, was dadurch ausgeglichen wird, daß die Zigarren keine Zigarren sind, aber der $\mathrm{Pa} ß \mathrm{mu}$ ein $\mathrm{Pa}$ sein, damit sie einen ins Land hereinlassen.“ (Bertolt Brecht: Prosa, Bd. 3, Berlin, Weimar 1973, S. 199.)
} 
Birus: Lessing und die Weltliteratur, S.18

und da angeben müsse, in der Prosa zu sehr auffallen dürfte. Auch erlaube, meinte ich, der Vers immer einen Absprung eher, wie ich ihn itzt zu meiner anderweitigen Absicht, bei aller Gelegenheit ergreifen muß. ${ }^{52}$

Sind so Stoffwahl, Stil und Versifikation des Nathan durchweg auf Lessings 'anderweitige - polemische - Absicht' beziehbar, so hat dieser theologiekritische Impetus andererseits in diesem „Dramatischen Gedicht“ keineswegs nur eine aus der Not geborene äußerliche (wenn man so will: journalistische) Einkleidung gefunden. Sondern Lessing ist damit in der Wechselbedingtheit von Form und Gehalt wohl das exemplarische Drama der europäischen Aufklärung und damit der nachhaltigste Beitrag der neueren deutschen Literatur vor Goethe zur Weltliteratur gelungen.

${ }^{52}$ An Karl Wilhelm Ramler, 18. 12. 1778, in: Lessing, FA XII, S. $214 \mathrm{f}$. 